\title{
Effects of zoledronic acid and the association between its efficacy and $\gamma \delta T$ cells in postmenopausal women with breast cancer treated with preoperative hormonal therapy: a study protocol
}

Eriko Sumi ${ }^{1 *}$, Tomoharu Sugie ${ }^{2}$, Kenichi Yoshimura ${ }^{3}$, Harue Tada $^{4}$, Takafumi Ikeda ${ }^{5}$, Eiji Suzuki ${ }^{6}$, Yoshimasa Tanaka ${ }^{7}$ Satoshi Teramukai ${ }^{8}$, Akira Shimizu ${ }^{5}$, Masakazu Toi ${ }^{6}$ and Nagahiro Minato ${ }^{9}$

\begin{abstract}
Background: Although the efficacy of zoledronic acid in postmenopausal women with breast cancer has been suggested, the underlying mechanism has not been fully clarified. Therefore, which patients may benefit from zoledronic acid and the optimal frequency of zoledronic acid administration are unclear. This study evaluates the effects of zoledronic acid on the tumor response in postmenopausal women with breast cancer and explores the relationship between its efficacy and $\gamma \delta T$ cells.

Methods/design: This study is an open-label, multi-institutional, single-arm, phase II clinical trial. Zoledronic acid will be administered once during preoperative hormonal therapy with letrozole for 24 weeks in postmenopausal women with Estrogen Receptor (ER)-positive, Human Epidermal Growth Factor Receptor 2 (HER2)-negative, clinical $\mathrm{T} 1$ or T2 NOMO breast cancer. The primary endpoint is the objective response rate measured by MRI at 12 and 24 weeks. The secondary endpoints are the associations between the frequency of $V \gamma 2 V \delta 2 T$ cells before the administration of zoledronic acid and the objective response, the association between the frequency of $V Y 2 V \delta 2 T$ cells and the Preoperative Endocrine Prognostic Index score, and the association between the frequency of V $22 \mathrm{~V} \delta 2$ T cells and Ki67 (MIB-1 index).
\end{abstract}

Discussion: This study is designed to determine the add-on effect of zoledronic acid during preoperative hormonal therapy and to investigate the changes of the frequency of $\mathrm{V} Y 2 \mathrm{~V} \delta 2 \mathrm{~T}$ cells after the administration of zoledronic acid to explore the potential mechanism of zoledronic acid in breast cancer patients.

Trial registration: This trial was registered at the UMIN Clinical Trials Registry as UMIN000008701.

Keywords: Zoledronic acid, Postmenopausal women, Breast cancer, $\gamma \delta$ T cells, Letrozole

\footnotetext{
* Correspondence: sumieri@kuhp.kyoto-u.ac.jp

'Department of Clinical Innovative Medicine, Institute for Advancement of Clinical and Translational Science, Kyoto University Hospital, 54 Shogoin Kawahara-cho, Sakyo-ku, Kyoto 606-8507, Japan

Full list of author information is available at the end of the article
} 


\section{Background}

According to the 2008 National Breast Cancer Registry Report [1], more than half of breast cancers are Estrogen Receptor (ER)-positive, Human Epidermal Growth Factor Receptor 2 (HER2)-negative, less than $5 \mathrm{~cm}$ in diameter and negative for lymph node metastasis. Nevertheless, treatment with paclitaxel and anthracycline-based combinations as an adjuvant therapy in ER-positive, HER2negative patients is less effective [2].

Hormonal therapy for early breast cancer has been shown to be effective [3]. Anastrozole is as effective as tamoxifen as a neoadjuvant treatment in postmenopausal women $[4,5]$. Letrozole is also more effective than tamoxifen as a preoperative treatment in postmenopausal patients with ER-and/or Progesterone Receptor (PgR)-positive primary breast cancer, although the response rate of $55 \%$ [6] is suboptimal.

Recently, the addition of zoledronic acid to adjuvant endocrine therapy in premenopausal women with breast cancer receiving goserelin improved disease-free survival and reduced the risk of disease-free survival events overall [7]. Moreover, the rates of both disease-free survival and overall survival were improved by the addition of zoledronic acid in postmenopausal patients [8,9].

Zoledronic acid appears to be efficacious in patients with early breast cancer [7-9] and in those with low levels of estrogen or a deficiency of estrogen [7-9] after menopause or oophorectomy and who were receiving hormonal therapy $[7,8]$.

The mechanism of the efficacy of zoledronic acid seems to be through direct anti-tumor effects [10]. Many theories and hypotheses have been presented on the mechanisms of zoledronic acid on tumor response [11-13]. The involvement of immune cells, such as $\mathrm{V} \gamma 2 \mathrm{~V} \delta 2 \mathrm{~T}$ cell (also called $\gamma \delta$ T cells), has also been suggested [14]. Zoledronic acid is one of the nitrogen-containing bisphosphonates (N-BPs). N-BPs, such as zoledronic acid and pamidronate, inhibit farnesyl diphosphate synthase, and its upstream metabolite diphosphomonoesters (e.g., isopentenyl diphosphate) accumulate in tumor cells. Then, $\mathrm{V} \gamma 2 \mathrm{~V} \delta 2 \mathrm{~T}$ cells specifically recognize and lyse the sensitized target tumor cells that bear or express antigenic substances $[15,16]$. The activated $\mathrm{V} \gamma 2 \mathrm{~V} \delta 2 \mathrm{~T}$ cells also secrete cytokines, such as interferon- $\gamma$ (IFN- $\gamma$ ) [17]. Although it was demonstrated that the frequency of $\gamma \delta$ T cells in peripheral blood was increased and that an acute-phase reaction occurred after treatment with pamidronate [18], the causal relationship between the function of $\gamma \delta$ T cells and the efficacy of pamidronate has not been elucidated.

The hypothesis in this study is that zoledronic acid will elicit an anti-tumor effect in patients with early breast cancer who have a high frequency of $\gamma \delta \mathrm{T}$ cells in the peripheral blood. In addition, the therapeutic roles of $\gamma \delta \mathrm{T}$ cells might be determined by measuring body temperature and IFN- $\gamma$ production because $\gamma \delta$ T cells secrete inflammatory cytokines in response to antigenic stimulation through their $\mathrm{T}$ cell receptors. The aim of this study is to examine the mechanism underlying the add-on effect of zoledronic acid and to determine whether the initial frequency of $\gamma \delta \mathrm{T}$ cells is related to the objective response rate of patients with early breast cancer who receive hormone therapy with zoledronic acid.

\section{Objectives}

The main objective of this study is to examine the addon effect of zoledronic acid in the treatment of postmenopausal women with ER-positive, HER2-negative breast cancer who receive preoperative hormonal therapy by letrozole. Other objectives are as follows: the determination of the frequency of $\mathrm{V} \gamma 2 \mathrm{~V} \delta 2 \mathrm{~T}$ cells in peripheral blood before the administration of zoledronic acid, the analysis of the associations between the frequency of $\mathrm{V} \gamma 2 \mathrm{~V} \delta 2 \mathrm{~T}$ cells before the administration of zoledronic acid, the objective response, the association between the frequency and Preoperative Endocrine Prognostic Index score, and the association between the frequency and Ki67 (MIB-1 index).

\section{Methods/design}

\section{Study design}

This study is an open-label, multi-institutional, singlearm, phase II clinical trial. A total of 75 women will be enrolled after they give written informed consent. The study was approved by the ethical committee at Kyoto University on October 18, 2012 (C-646) and will be approved by the ethical committees at the participating hospitals prior to study initiation at each site. The study protocol complies with the Helsinki declaration [19] and the Ethics Guidelines for Clinical Research of the Ministry of Health, Labor, and Welfare [20]. This study was registered with the UMIN Clinical Trials Registry as UMIN000008701 (http://www.umin.ac.jp/ctr/index.htm). An additional study to investigate tumor infiltration with $\gamma \delta \mathrm{T}$ cells at baseline, 12 weeks and 24 weeks was also approved by the ethical committee at Kyoto University on June 1, 2013 (E1723).

\section{Eligibility criteria}

Women will be included in the study if they meet the following criteria: have primary cT1-2, NOM0 breast cancer; histologically confirmed invasive ductal cancer; ER-positive and HER2-negative (i.e., as determined by either immunohistochemistry (IHC) or by FISH/DISH (fluorescence in situ hybridization/Dual Color in situ Hybridization)) breast cancer; low levels or a deficiency of estrogen (i.e., women aged 60 years or older that have been postmenopausal for more than 4 years or have had a post-bilateral oophorectomy); no prior treatment for 
breast cancer; measurable disease on enhanced MRI within 6 weeks prior to study entry; an Eastern Cooperative Oncology Group (ECOG) performance score of 0 or 1; are within the age range of 20 to 75; have adequate renal function with a Ccr (Cockcroft-Gault) $>=30 \mathrm{~mL} /$ min; and have adequate hepatic function with serum bilirubin $<=2.0$ and AST $/$ ALT $<=100$.

Women will be excluded if they have: hyperparathyroidism that requires management; uncontrolled diabetes mellitus; diseases that require continuous management with systemic corticosteroids; dental and/or periodontal disease that requires invasive treatment after enrollment; currently active or past malignancies within the past five years; prior bisphosphonate therapy, if they have undergone hormone replacement therapy within 7 days prior to enrollment; are hypersensitive to contrast materials for MRI; or used other investigational drugs within 28 days prior to enrollment.

\section{Intervention}

Participants will take $2.5 \mathrm{mg} /$ day of letrozole orally within 7 days of enrollment in the study. Participants will receive a single-drip injection of zoledronic acid on day 28. The dose of zoledronic acid will be reduced when the participants demonstrate impaired renal function (i.e., when the Ccr is equal to or less than $60 \mathrm{~mL} / \mathrm{min}$ ) according to Table 1. A single administration of zoledronic acid is expected to be effective because frequent zoledronic acid treatments lead to a decrease in blood $\mathrm{V} \gamma 2 \mathrm{~V} \delta 2 \mathrm{~T}$ cell levels [14]. Cytokines such as IL-2 are not used in combination with zoledronic acid because patients with early breast cancer without any previous treatment have a potential to restore immune responses against tumor antigens without administration of cytokines such as IL-2. Participants will take letrozole every day for 24 to 26 weeks until they have a mastectomy as a part of routine medical care. The study treatment will be terminated upon disease progression, episodes of serious adverse events, or in patients for whom zoledronic acid cannot be administered within 35 days of starting letrozole treatment.

\section{Measurements}

Patients will be evaluated clinically and through laboratory testing, radiological testing and pathological testing, according to Table 2.

The primary endpoint is the objective response rate (ORR). The ORR is defined as the proportion of the complete response $(\mathrm{CR})$ and the partial response (PR) within

Table 1 Dose of zoledronic acid

\begin{tabular}{lllll}
\hline & \multicolumn{4}{l}{ Ccr $(\mathbf{m L} / \mathbf{m i n})$} \\
\cline { 2 - 5 } & $>\mathbf{6 0}$ & $\mathbf{5 0 - 6 0}$ & $\mathbf{4 0 - 4 9}$ & $\mathbf{3 0 - 3 9}$ \\
\hline Dose of zoledronic acid & $4 \mathrm{mg}$ & $3.5 \mathrm{mg}$ & $3.3 \mathrm{mg}$ & $3.0 \mathrm{mg}$ \\
\hline
\end{tabular}

the best overall response based on MRI evaluation. Patients will undergo tumor assessments at baseline, 12 weeks and 24 weeks by investigators using the Response Evaluation Criteria in Solid Tumors (RECIST) version 1.1 [21]. The imaging modality is restricted to MRI because MRI is superior to other modalities for the detection and staging of invasive breast cancer, and MRI measurements after neoadjuvant treatment are more highly correlated with pathological tumor size than mammography or ultrasound [22]. The secondary endpoints are the change in tumor volume, ORRs using ultrasonography or clinical assessment, breast-conserving surgery, the frequency of Vy2V82 T cells, PEPI score [23], Ki67 (MIB-1 index) and safety.

Tumor volume will be measured using MRI volumetry at baseline, 12 weeks and 24 weeks by an independent committee. The contrast-enhanced MRI will be performed according to the European Society of Breast Imaging (EUSOBI) guidelines [24]. ORRs using ultrasonography or clinical assessment will be evaluated separately by the investigators according to RECIST. A partial resection of the breast will be regarded as breast-conserving surgery after the completion of letrozole treatment.

The frequency of $\mathrm{V} \delta 1^{+} \mathrm{T}$ cells and $\mathrm{V} \delta 2^{+} \mathrm{T}$ cells among $\mathrm{CD}^{+} \mathrm{T}$ cells in peripheral blood mononuclear cells will be analyzed by two-color flow cytometry [11] and will be evaluated at baseline, 4 weeks and 20 weeks after the administration of zoledronic acid.

Peripheral mononuclear cells (PBMC) will be purified by density gradient centrifugation. For flow cytometric experiments, $2 \times 10^{5}$ cells of PBMC in each well will be stained with mAbs. The following mAbs will be used: fluorescein isothiocyanate (FITC)-conjugated anti- V $\delta 1 \mathrm{mAbs}$ (Thermo Scientific, Rockford, IL), V 22 mAbs (Beckman Coulter Inc., Fullerton, CA), anti-CD3 mAbs (BioLegend, San Diego, CA), phycoerythrin (PE)-conjugated anti-CD3, CD4, CD8, CD25, NKG2D mAbs (BD Biosciences, San Diego, CA) and FcR blocking reagent (Miltenyi Biotec $\mathrm{GmbH}$, Bergisch Gradbach, Germany) will be used. The stained cells will be subjected to 2-color flow cytometry using a FACSCalibur flow cytometer (Becton-Dickinson, Franklin Lakes, NJ) and the analyses will be performed on a single sample. The serum at 5 hours after administration of zoledronic acid will be collected and subjected to ELISA for IFN- $\gamma$ level using an ELISA Kit (PEPROTECH, Rocky Hill, NJ) according to the manufacturer's instructions. The analyses will be conducted on triplicate samples.

Adverse events will be evaluated according to the Common Terminology Criteria for Adverse Events (CTCAE) version 4.0 [25].

\section{Data analysis}

The sample size was determined using the Bayesian method based on predictive distributions [26,27]. The ORR 
Table 2 Trial schedule

Protocol treatments and assessments

Letrozole

Pre-enrollment

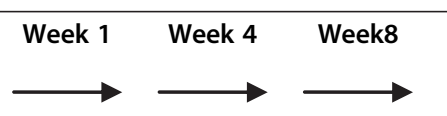

Week 12

Week 16

Week 20 Week 24 Mastectomy

Protocol treatments

Letrozole

Zoledronic acid

$\downarrow$

Demographics, current medications, dental examination

Physical examination

Complete blood count, blood biochemical test, bone metabolism markers

Enhanced MRI, tumor markers

Palpation, ultrasound examination

$V \delta 1+T$ cells and $V \delta 2+T$ cells in peripheral blood ${ }^{3}$

IFN- $\gamma^{4}$

Pathological examination ${ }^{5}$

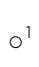

○

$\circ$

$\circ$

PEPI score

'Body temperature before and 4-6 hours after the administration of zoledronic acid.

${ }^{2}$ Bone metabolism markers that are expected.

${ }^{3}$ The frequency of $\mathrm{V} \delta 1+\mathrm{T}$ cells and $\mathrm{V} \delta 2+\mathrm{T}$ cells among CD3+ T cells in peripheral blood.

${ }^{4}$ IFN- $\gamma$ at 4-6 hours after the administration of zoledronic acid.

${ }^{5} \mathrm{ER}, \mathrm{PgR}, \mathrm{Ki} 67$. 
of preoperative hormonal therapy by letrozole is estimated to be $45 \%$ [28]. The threshold ORR of $45 \%$ was used as the prespecified target value for the primary endpoint. The uniform distribution and the degenerated distribution at an expected ORR of $60 \%$ were used as the analysis prior and the design prior, respectively. The sample size of 75 is required to preserve the Bayesian power of $80 \%$ with a prespecified probability threshold of $95 \%$ to declare the treatment efficacious.

The primary statistical analysis will be performed using the Bayesian approach according to the intention-to-treat principle. The posterior beta distribution of the ORR will be calculated using the prior uniform distribution. If the posterior probability that the ORR is greater than the prespecified target value of $45 \%$ exceeds a prespecified probability threshold of $95 \%$, the treatment will be considered efficacious.

A subset analyses for the ORR based on MRI and tumor volume using MRI volumetry according to (a) equal and greater or less than the median of the frequency of $\mathrm{V} \gamma 2 \mathrm{~V} \delta 2 \mathrm{~T}$ cells before the administration of zoledronic acid, (b) equal and greater or less than the median of IFN- $\gamma$ at 5 hours after the administration of zoledronic acid and (c) presence of fever and/or flu-like symptoms, are planned. Exploratory subgroup analyses for the frequency of $\mathrm{V} \gamma 2 \mathrm{~V} \delta 2 \mathrm{~T}$ cells according to (a) equal and greater or less than a body mass index of 25 , (b) $0,1-3$, or over 3 of PEPI score, (c) equal and greater or less than 14 of Ki67, (d) equal and greater or less than the median of IFN- $\gamma$ at 5 hours after the administration of zoledronic acid and (e) presence of fever and/or flu-like symptoms, are also planned.

\section{Discussion}

We presented the design of a single-arm phase II study to evaluate the add-on effect of zoledronic acid in preoperative hormonal therapy. The single-arm design will not clarify whether the frequency of $\mathrm{V} \gamma 2 \mathrm{~T}$ cells before administration of zoledronic acid influences the outcome of breast cancer patients regardless of zoledronic acid or if they influence the outcome related to administration of zoledronic acid. Nevertheless, the changes of the frequency of $\mathrm{V} \gamma 2 \mathrm{~V} \delta 2 \mathrm{~T}$ cells after the administration of zoledronic acid suggest the potential immunological mechanism of zoledronic acid in breast cancer patients.

\section{Trial status}

Ongoing.

\section{Abbreviations}

ER: Estrogen receptor; HER2: Human epidermal growth factor receptor 2; N-BPS: Nitrogen-containing bisphosphonates; IFN- $\gamma$ : Interferon- $\gamma$; PEPI: Preoperative endocrine prognostic index; IHC: immunohistochemistry; FISH: Fluorescence in situ hybridization; DISH: Dual color in situ Hybridization;
ECOG: Eastern cooperative oncology group; ORR: Objective response rate; CR: Complete response; PR: Partial response; RECIST: Response evaluation criteria in solid tumors; EUSOBI: European society of breast imaging; CTCAE: Common terminology criteria for adverse events.

\section{Competing interests}

MT, TS and AS were awarded industry grants from Novartis Pharmaceuticals, Japan to conduct this trial. TS received speaker honoraria from Novartis

Pharmaceuticals.

\section{Authors' contributions}

ES participated in the coordination of the trial and drafted the protocol. TS, ES and MT conceptualized the study. YT and NM participated in the immunological review. HT participated in the data collection. KY participated in the study design, the statistical review and drafted the manuscript. TI and AS participated in the coordination of the trial. ST participated in the study design and the statistical review. All authors read and approved the final manuscript.

\section{Acknowledgements}

This work was supported by the "Coordination, Support, and Training Program for Translational Research" and the "Translational Research Network Program" of the Ministry of Education, Science, Culture, Sports, and Technology of Japan (awarded to MT, TS, ES, NM and AS).

\section{Author details}

'Department of Clinical Innovative Medicine, Institute for Advancement of Clinical and Translational Science, Kyoto University Hospital, 54 Shogoin Kawahara-cho, Sakyo-ku, Kyoto 606-8507, Japan. ${ }^{2}$ Breast Surgery, Kansai Medical University Hirakata Hospital, Osaka, Japan. ${ }^{3}$ Center for Clinical Research, Kobe University Hospital, Hyogo, Japan. ${ }^{4}$ Department of Data Science, Institute for Advancement of Clinical and Translational Science, Kyoto University Hospital, Kyoto, Japan. ${ }^{5}$ Department of Experimental Therapeutics, Institute for Advancement of Clinical and Translational Science, Kyoto University Hospital, Kyoto, Japan. ${ }^{6}$ Department of Breast Surgery, Kyoto University Hospital, Kyoto, Japan. ${ }^{7}$ Center for Therapeutic Innovation, Graduate School of Biomedical Sciences, Nagasaki University, Nagasaki, Japan. ${ }^{8}$ Department of Biostatistics, Graduate School of Medical Science, Kyoto Prefectural University of Medicine, Kyoto, Japan. ${ }^{9}$ Department of Immunology and Cell Biology, Graduate School of Medicine, Kyoto University, Kyoto, Japan.

Received: 22 June 2014 Accepted: 22 October 2014

Published online: 25 November 2014

\section{References}

1. The Japanese Breast Cancer Society: National Breast Cancer Registry Report 2008. [http://www.jbcs.gr.jp/people/nenjihoukoku/2008nenji.pdf]

2. Martin M, Rodriguez-Lescure A, Ruiz A, Alba E, Calvo L, Ruiz-Borrego M, Munarriz B, Rodriguez CA, Crespo C, De Alava E, Lopez Garcia-Asenjo JA, Guitian MD, Almenar S, Gonzalez-Palacios JF, Vera F, Palacios J, Ramos M, Gracia Marco JM, Lluch A, Alvarez I, Segui MA, Mayordomo Jl, Anton A, Baena JM, Plazaola A, Modolell A, Pelegri A, Mel JR, Aranda E, Adrover E, et al: Randomized phase 3 trial of fluorouracil, epirubicin, and cyclophosphamide alone or followed by Paclitaxel for early breast cancer. J Nat/ Cancer Inst 2008, 100(11):805-814.

3. Early Breast Cancer Trialists' Collaborative G: Effects of chemotherapy and hormonal therapy for early breast cancer on recurrence and 15-year survival: an overview of the randomised trials. Lancet 2005, 365(9472):1687-1717.

4. Smith IE, Dowsett M, Ebbs SR, Dixon JM, Skene A, Blohmer JU, Ashley SE, Francis S, Boeddinghaus I, Walsh G: Neoadjuvant treatment of postmenopausal breast cancer with anastrozole, tamoxifen, or both in combination: the Immediate Preoperative Anastrozole, Tamoxifen, or Combined with Tamoxifen (IMPACT) multicenter double-blind randomized trial. J Clin Oncol 2005, 23(22):5108-5116.

5. Cataliotti L, Buzdar AU, Noguchi S, Bines J, Takatsuka Y, Petrakova K, Dube P, de Oliveira CT: Comparison of anastrozole versus tamoxifen as preoperative therapy in postmenopausal women with hormone receptor-positive breast cancer: the Pre-Operative "Arimidex" Compared to Tamoxifen (PROACT) trial. Cancer 2006, 106(10):2095-2103. 
6. Eiermann W, Paepke S, Appfelstaedt J, Llombart-Cussac A, Eremin J, Vinholes J, Mauriac L, Ellis M, Lassus M, Chaudri-Ross HA, Dugan M, Borgs M, Letrozole Neo-Adjuvant Breast Cancer Study Group: Preoperative treatment of postmenopausal breast cancer patients with letrozole: a randomized double-blind multicenter study. Ann Oncol 2001, 12(11):1527-1532.

7. Gnant M, Mlineritsch B, Stoeger H, Luschin-Ebengreuth G, Heck D, Menzel C, Jakesz R, Seifert M, Hubalek M, Pristauz G, Bauernhofer T, Eidtmann H, Eiermann W, Steger G, Kwasny W, Dubsky P, Hochreiner G, Forsthuber EP, Fesl C, Greil R, Austrian Breast Colorectal Cancer Study Group, Vienna Austria: Adjuvant endocrine therapy plus zoledronic acid in premenopausal women with early-stage breast cancer: 62-month follow-up from the ABCSG-12 randomised trial. Lancet Oncol 2011, 12(7):631-641.

8. Coleman R, de Boer R, Eidtmann H, Llombart A, Davidson N, Neven P, Von Minckwitz G, Sleeboom HP, Forbes J, Barrios C, Frassoldati A, Campbell I, Paija O, Martin N, Modi A, Bundred N: Zoledronic acid (zoledronate) for postmenopausal women with early breast cancer receiving adjuvant letrozole (ZO-FAST study): final 60-month results. Ann Oncol 2013, 24(2):398-405.

9. Coleman RE, Marshall H, Cameron D, Dodwell D, Burkinshaw R, Keane M, Gil M, Houston SJ, Grieve RJ, Barrett-Lee PJ, Ritchie D, Pugh J, Gaunt C, Rea U, Peterson J, Davies C, Hiley V, Gregory W, Bell R: Breast-cancer adjuvant therapy with zoledronic acid. N Engl J Med 2011, 365(15):1396-1405.

10. Coleman RE, Winter MC, Cameron D, Bell R, Dodwell D, Keane MM, Gil M, Ritchie D, Passos-Coelho JL, Wheatley D, Burkinshaw R, Marshall SJ, Thorpe H, Azure Investigators: The effects of adding zoledronic acid to neoadjuvant chemotherapy on tumour response: exploratory evidence for direct anti-tumour activity in breast cancer. Br J Cancer 2010, 102(7):1099-1105.

11. Mundy GR: Metastasis to bone: causes, consequences and therapeutic opportunities. Nat Rev Cancer 2002, 2(8):584-593.

12. Green JR, Guenther A: The backbone of progress-preclinical studies and innovations with zoledronic acid. Crit Rev Oncol Hematol 2011, 77(Suppl 1):S3-S12.

13. Winter MC, Holen I, Coleman RE: Exploring the anti-tumour activity of bisphosphonates in early breast cancer. Cancer Treat Rev 2008, 34(5):453-475.

14. Sugie T, Murata-Hirai K, Iwasaki M, Morita CT, Li W, Okamura H, Minato N, Toi M, Tanaka Y: Zoledronic acid-induced expansion of gammadelta T cells from early-stage breast cancer patients: effect of IL-18 on helper NK cells. Cancer Immunol Immunother 2013, 62(4):677-687.

15. Tanaka Y, Morita CT, Tanaka Y, Nieves E, Brenner MB, Bloom BR: Natural and synthetic non-peptide antigens recognized by human gamma delta $T$ cells. Nature 1995, 375(6527):155-158.

16. Tanaka $Y$ : Human gamma delta $T$ cells and tumor immunotherapy. J Clin Exp Hematop 2006, 46(1):11-23.

17. Miyagawa F, Tanaka Y, Yamashita S, Minato N: Essential requirement of antigen presentation by monocyte lineage cells for the activation of primary human gamma delta T cells by aminobisphosphonate antigen. J Immunol 2001, 166(9):5508-5514.

18. Kunzmann V, Bauer E, Wilhelm M: Gamma/delta T-cell stimulation by pamidronate. N Engl J Med 1999, 340(9):737-738.

19. The World Medical Association: WMA Declaration of Helsinki - ethical principles for medical research involving human subjects. [http://www. wma.net/en/30publications/10policies/b3/17c.pdf]

20. The Ministry of Health, Labor, and Welfare: ethics guidelines for clinical research. [http://www.mhlw.go.jp/general/seido/kousei/i-kenkyu/ index.html]

21. Eisenhauer EA, Therasse P, Bogaerts J, Schwartz LH, Sargent D, Ford R, Dancey J, Arbuck S, Gwyther S, Mooney M, Rubinstein L, Shankar L, Dodd L, Kaplan R, Lacombe D, Verweij J: New response evaluation criteria in solid tumours: revised RECIST guideline (version 1.1). Eur J Cancer 2009, 45(2):228-247.

22. Fumagalli D, Bedard PL, Nahleh Z, Michiels S, Sotiriou C, Loi S, Sparano JA, Ellis M, Hylton N, Zujewski JA, Hudis C, Esserman L, Piccart M: A common language in neoadjuvant breast cancer clinical trials: proposals for standard definitions and endpoints. Lancet Oncol 2012, 13(6):e240-e248.

23. Ellis MJ, Tao Y, Luo J, A'Hern R, Evans DB, Bhatnagar AS, Chaudri Ross HA, von Kameke A, Miller WR, Smith I, Eiermann W, Dowsett M: Outcome prediction for estrogen receptor-positive breast cancer based on postneoadjuvant endocrine therapy tumor characteristics. J Nat/ Cancer Inst 2008, 100(19):1380-1388.
24. Breast MRI: guidelines from the European Society of Breast Imaging. [http://www.eusobi.org/cms/website.php?id=/en/society/guidelines.htm]

25. The Japanese Clinical Oncology Group: Japanese version of the Common Terminology Criteria for Adverse Events version 4.0. [http://www.jcog.jp/doctor/tool/ctcaev4.html]

26. Sambucini V: A Bayesian predictive two-stage design for phase II clinical trials. Stat Med 2008, 27(8):1199-1224.

27. Teramukai S, Daimon T, Zohar S: A Bayesian predictive sample size selection design for single-arm exploratory clinical trials. Stat Med 2012, 31(30):4243-4254.

28. A randomized study of adjuvant endocrine therapy with or without chemotherapy for postmenopausal breast cancer patients who responded to neoadjuvant letrozole: An interim efficacy analysis of the new primary endocrine-therapy origination study (NEOS / N-SAS BC06). [http://sabcs.posterview.com/]

doi:10.1186/s12967-014-0310-2

Cite this article as: Sumi et al:: Effects of zoledronic acid and the association between its efficacy and $\gamma \delta T$ cells in postmenopausal women with breast cancer treated with preoperative hormonal therapy: a study protocol. Journal of Translational Medicine 2014 12:310.

\section{Submit your next manuscript to BioMed Central and take full advantage of:}

- Convenient online submission

- Thorough peer review

- No space constraints or color figure charges

- Immediate publication on acceptance

- Inclusion in PubMed, CAS, Scopus and Google Scholar

- Research which is freely available for redistribution

Submit your manuscript at www.biomedcentral.com/submit
C) Biomed Central 